\title{
A Social Network Information Dissemination Model Based on Evolutionary Game Considering Node's Attitude
}

\author{
Hongcheng Huang ${ }^{1}$, Licheng $\mathrm{Lai}^{2}$, Min $\mathrm{Hu}^{3}$,Mengyuan Dong ${ }^{4}$, Tingting Wang ${ }^{5}$ \\ \{huanghc@cqupt.edu.cn ${ }^{1}$, Lai_licheng@163.com ${ }^{2}$, humin@cqupt.edu.cn ${ }^{3}$ \} \\ School of Communication and Information Engineering, Chongqing University of Posts and \\ Telecommunications $1,2,3,4,5$
}

\begin{abstract}
As a kind of social attribute of bounded rational users, attitude continuously selects and updates in the process of interaction between nodes, which makes the behavioral game between communication subjects and affects the dissemination of information. To analyze the impact of attitude changes on information, this paper proposes an information propagation model based on evolutionary game. Firstly, from the individual point of view, the update rules of the node's attitude are defined according to non-Bayesian social learning rules. Secondly, the game matrix between nodes based on attitude value is established, and the information dissemination model based on node attitude is established according to the evolutionary analysis paradigm. By replicating the equilibrium solution of dynamic equations with both positive and negative attitudes, and analyzing the stability of the corresponding equilibrium points, the evolution mechanism of dynamic interaction between attitude nodes is obtained. Finally, the impact of different attitudes on information dissemination was analyzed by combining SIS model. The validity of the proposed model is verified by numerical analysis and simulation experiments, and the simulation results show that the change of different attitude of nodes plays an important role in information dissemination.
\end{abstract}

Keywords: social network; information dissemination; evolutionary game; node attitude.

\section{Introduction}

Since entering the 21st century, the rapid development of the Internet has also led to the development of various social software, such as Weibo, WeChat, Twitter and so on. Social software has brought great convenience to people's lives, and people's way of information exchange has become more and more rich, forming a rich and colorful social network world. The birth of social networks has galvanized a change in the way of information dissemination. The information continues to diffuse and spread in the network due to people's daily social activities, and the information dissemination of social network has the characteristics of shortterm explosiveness and fast propagation for hotspot information [1-4]. Today, the number of social network users is huge, so that the scope of information dissemination is more extensive and the impact is also greater. Therefore, the research on the information dissemination of social networks is an important subject, which has attracted the attention of many scholars, and it is of great significance to study the information dissemination model, which can make 
corresponding measures for the control of follow-up information and the guidance of public opinion.

Scholars have done a lot of research on the information dissemination of social networks. In the field of biology, the spread of the virus mainly contains three stages: the susceptible body, the infective body and the immune body, which indicates that a infected cell will soon affect other individuals and may change their original state. According to this similar law, scholars model the information dissemination process of social networks, dividing the node state in the network into three stages: the susceptible node that does not receive the information, but will propagate when the information is received; the infective node that has received the message and will propagate the message; and finally the immune node that receives the information but does not propagate the information. The information dissemination model of social network currently established on this basis has the SI (susceptible-infected) [5] model, the SIS (susceptible-infected-susceptible) [6] model, and the SIR ( susceptible-infected-recovered) model [7]. In the process of information dissemination, the immune state node will also become a propagation node over time, which means the immune node will have an incubation period, so some researchers proposed the SIRS model [8]. This kind of models the propagation trend from the macroscopic point of view, which neglects the influence of other factors on the information dissemination. These influencing factors include the individual's psychology, the social environment, the nature of the information itself and so on. Therefore, the actual propagation process of information cannot be well described. So the researchers further study the dissemination of information, some scholars study with the network topology and community structure. In [9], from the perspective of network structure, authors considered how the changes in community structure and personal interests impact on information dissemination. By establishing the information dissemination model considering the factors of social reinforcement and time attenuation, it is shown that the high connection degree of community structure will speed up the propagation of information, and the scope of information dissemination will be greatly reduced when the value of individual benefit is decreased. In [10], this paper analyzed the influence of the content nature of the information on the propagation, and puts forward a new model based on the SIR model: the SICR (susceptible-infected-counterattack-refractory) model. It took into account the rumor counterattack state node, and used the average field equation to describe the evolution process of rumor information in homogeneous network. Considering the influence of nodes in social networks, Xiao and others authors [11] combined the SIR Model and social influence factors to extract the user's personal memory and historical interaction records from two aspects of network topology and user behavior. Then, they proposed a method to measure the social influence of users and verified that the proposed model can effectively perceive the information evolution situation of social network. This paper in [12] further considered the influencing factors of node feedback information, and analyzed the characteristics of social network and the social attributes of communicators, in which authors adopted a new negative feedback mechanism of social network information, defined the feedback principle of feedback function description information, and constructed the evolution propagation tree of information to reveal the complex interaction in the user relationship, social communities and cyberspace information in social network.

Although the existing researches take different factors into account for the dissemination of information, they ignore the influence of node's attitude on the communication of information in the process of social network dissemination. The willingness of different users to propagate information depends on their own attitude, while the nodes of different attitudes may be affected by the neighbor node in the process of interaction. The attitude choice of 
information in the process of information dissemination makes the interaction between the communication subjects, which results in the behavior game between information dissemination. In order to describe the influence of this dynamic interaction process on information dissemination, this paper proposes a information dissemination model of social network based on evolutionary game considering the attitude of nodes. From the individual point of view, according to the non-Bayesian social learning rules to define the attitude of nodes and update rules. We choose the strategy based on the game between active nodes and negative nodes, establish an information dissemination model based on node attitude, and analyze the influence of different attitudes on information dissemination.

The various chapters of this paper are arranged as follows: The first section introduces the research background and the current research status at home and abroad. The second section mainly introduces the attitude interaction process between nodes in social networks. And the third section proposes an evolutionary game model of information dissemination based on node attitude. In the fourth section, numerical analysis and simulation experiments are carried out on the proposed model to verify the validity of the model. The final section analyzes and summarizes the full text.

\section{Analysis of attitude interaction between nodes in social networks}

Due to the value of information, the user's psychology and the environment state and other influencing factors, it is possible to influence the user node's willingness to spread the information, which means the user's attitude value has an important influence on the information dissemination. The attitude of the user node is divided into two states: the positive attitude of the node and the negative attitude of the node. We assume that its attitude value is $\mu$, and the definition of its value is as follows:

$$
\mu \begin{cases}>0 & \text { Positive attitude node } \\ <0 & \text { Negative attitude node }\end{cases}
$$

Among them, if the attitude value is positive, the greater the value, the more active the attitude, and the more likely it is to promote the spread of the message. On the contrary, if the attitude value is in negative interval, the smaller the value, the lower the willingness of its nodes to propagate information, and the more likely it is to inhibit the spread of information. When the node receives the message from the neighbor node, the attitude interaction game between the nodes may change the attitude value of the two sides to the information dissemination. Assuming that the positive node increases the attitude value of the negative attitude node, the probability of the negative node chooses to propagate the information increases. Similarly, the negative attitude node reduces the attitude of the positive attitude node, then the positive node will reduce the probability of the message propagation that means the interaction result has two kinds of results to propagate or not propagate the information. We respectively assume that the positive attitude node chooses to propagate the information probability is $\lambda$ and the negative attitude node chooses not to propagate the message probability is $\gamma$. For information of social networks, the proportion of information on the network is $\lambda(1-\gamma)$, and the proportion of non-propagation is $\gamma(1-\lambda)$,before the nodes interact. So the probability of a coordinated attitude towards information dissemination is the sum of the two, that is: 


$$
\begin{aligned}
\phi & =\lambda(1-\gamma)+\gamma(1-\lambda) \\
& =\lambda+\gamma-2 \lambda \gamma
\end{aligned}
$$

Among them, the values of $\lambda$ and $\gamma$ are both $[0,1]$.

For the information in the social network, if it's positive and the positive node can make the negative node change attitude to spread the information, it will be conducive to the dissemination of positive energy information. And for rumors and other false information, if negative nodes can reduce the positive node attitude value, it will be conducive to inhibiting the random spread of rumors. As can be known from the formula (1), the value of $\phi$ is about plane $\lambda+\gamma=1$ symmetry. When $\phi$ obtains the maximum value 1 , values of $\lambda$ and $\gamma$ are $(1,0)$ and $(0,1)$. At this time, it indicates that either the node tends to propagate information, or it tends not to propagate the information. In the process of interactive game between positive attitude node and negative attitude node, the two affect each other. For the value of $(\lambda, \gamma)$ corresponding to the physical meaning represented in the game, the corresponding value and the meaning express as shown in table 1 , where $\mathrm{x}$ and $\mathrm{y}$ in table 1 , represent that and take any value in $[0,1]$.

Table 1. The meaning of information propagation corresponding to the value of $(\lambda, \gamma)$

\begin{tabular}{cc}
\hline$(\lambda, \gamma)$ & Meaning of the different values of $(\lambda, \gamma)$ \\
\hline$(1,0)$ & $\begin{array}{c}\text { Positive nodes change the willingness of negative attitude nodes to spread } \\
\text { and promote the dissemination of information }\end{array}$ \\
$(0,1)$ & $\begin{array}{c}\text { Negative nodes change the willingness of positive attitude nodes to spread } \\
\text { and inhibit the dissemination of information }\end{array}$ \\
$(1,1)$ & Neither side has changed the other's willingness to spread. \\
$(0,0)$ & Both sides make each other change their willingness to spread at the same \\
$(1, \mathrm{x})$ & time
\end{tabular}

As can be seen from the table1., when the value of $(\lambda, \gamma)$ is $(1,1)$ or $(0,0)$, they respectively express the results of the two game states, namely neither side has changed the attitude of the other side, or the two sides exchanged their original attitude to choose the attitude of the other side. At this time the value of $\phi$ is 0 , it indicates that the probability of such a situation is 0 . Combining with the above meaning of information dissemination, we establish an evolutionary game model based on information dissemination of the positive and negative attitude of nodes. The complete rationality of the individual is considered in the traditional game model. Unlike the traditional game, the user in the realistic social network, is not completely rational but the finite rational individual. In [13], the evolution game combines the game theory with the dynamic evolution process, and the communication of information in the network changes with the change of time. The individual's information dissemination behavior adjusts the attitude dynamically in the process of interacting with the neighbor node, and the finite rationality of the evolutionary game expresses the individual's self-regulation ability. In the game process, according to the other person's experience and learning the other person's behavior, we dynamically look for the better strategy to achieve a stable evolution state finally. 


\section{Evolutionary Game Model of Information Propagation Based on Node Attitude}

\subsection{Node attitude update rules and related definitions}

The main body of social network is the reality of the crowd users, social learning research found that users have their own attitude toward whether information on the social network is forward, and their attitude will be changed according to different information and different influencing factors. After generating an initial attitude towards a message, it may adjust its attitude after interacting with surrounding users [14]. The non-Bayesian learning method of social learning is that the individual carries on the behavior learning through the imitation reproduction learning principle, can embody the limited rational behavior of the user in the social network. Therefore, this paper describes the attitude update rules of nodes through nonBayesian learning method. Assuming that node $j$ in the network propagates messages to node $i$ at $t+1$ time, and the attitude values of nodes $i$ and $j$ at the previous moment are $\mu_{t}^{i}$ and $\mu_{t}^{j}$ respectively, at this time, according to the non-Bayesian social learning model, the attitude of the nodes is updated to

$$
\mu_{t+1}^{i}=\mu_{t}^{i}+\left(\mu_{t}^{j}-\mu_{t}^{i}\right) \zeta(i, j)
$$

In the formula, $\zeta(i, j)$ represents the convergence parameter of the attitude of node $i$ (or $j$ ) to $j$ (or $i$ ), which is described as the rate of change of the attitude of the node $i$ (or $j$ ), and the range of convergence parameters is $[0,1]$.

For the positive or negative attitude node in the network, after receiving the information to interact between the neighbor nodes, if the two sides of the game after the attitude changes, then follow the above update rules, and make the attitude of the other party changes will get the corresponding benefits. Therefore, we make the following definitions :

Definition 1: The Inter-node evolutionary game model of different attitudes can be represented as four tuples $M o d e l=(N, S, P, E)$, of which:

1) $N=\left(N_{\text {active }}, N_{\text {negative }}\right)$ represents a set of nodes involved in a game, the former representing a set of positive attitude nodes, and the latter representing a collection of negative attitude nodes.

2) $S=$ (dissmination, non-dissemination) represents a set of strategies and states for game participants, where both positive and negative attitudes are two strategies, namely, the dissemination and non-dissemination of information.

3) $P=(\lambda, \gamma)$ represents the probability set of the policy selection of the attitude node, where $\lambda$ indicates the probability that the positive attitude node chooses to propagate the message, and $\gamma$ indicates the probability that the negative attitude node does not propagate the information..

4) $E=\left(E_{\text {active }}, E_{\text {negative }}\right)$ represents the expected value of positive and negative attitude nodes obtained during the game.

In the actual attitude node game process, we discuss that the positive attitude node makes the attitude of the negative node to spread the information tendency. As the information 
dissemination communicator, when the information dissemination communicator succeeds in causing other nodes to have a willingness to disseminate the information, they will be inspired, so that other nodes also propagate the information. At this point the active node will be rewarded, and the return of its attitude value is $R$, so the positive node attitude value updated to $\mu_{t}^{i}+R$, the negative node attitude value is updated to $\mu_{t+1}^{j}=\mu_{t}^{j}+\left(\mu_{t}^{i}-\mu_{t}^{j}\right) \zeta(i, j)$ according to the previous rules, and there is no additional attitude value gain. In order to facilitate the expression, $\mu_{t+1}^{j}$ is representd by $K$. Similarly, when the negative attitude node makes the positive nodes to the willingness to not spread the information, as the information dissemination inhibitor, successfully inhibits the positive node propagation intention after the self-benefit is $J$, here $J$ is aminus value, which indicates the negative attitude node's attitude value further decreases, and is more likely to suppress the information dissemination. Therefore, the negative node attitude updates to the $\mu_{t}^{j}+J$, positive attitude node's attitude value updated to $\mu_{t+1}^{i}=\mu_{t}^{i}+\left(\mu_{t}^{j}-\mu_{t}^{i}\right) \zeta(i, j)$. For convenience, this formula is represented by the letter $M$. When both the positive attitude node and the negative attitude node do not change the other person's willingness to spread, the attitude value is the initial attitude value. If the other person's willingness to spread is changed at the same time, the attitude of both parties is updated according to the non-Bayesian update rule, and the same benefit is obtained, so the positive node attitude value of the income is $M+V$. In this paper, the negative attitude node attitude value expressed in minus value, so its attitude gain is $K-V$. In summary, the attitude yield matrix of the nodes is shown in table 2 :

Table 2. Game matrix based on node attitude value

\begin{tabular}{lcc}
\hline Positivelnegative & dissemination & Non-dissemination \\
\hline $\begin{array}{c}\text { dissemination } \\
\text { Non- }\end{array}$ & $\left(\mu_{t}^{i}+R, K\right)$ & $\left(\mu_{t}^{i}, \mu_{t}^{j}\right)$ \\
dissemination & $(M+V, K-V)$ & $\left(M, \mu_{t}^{j}+J\right)$ \\
\hline
\end{tabular}

\subsection{The dynamic equation structure of node attitude interaction evolution}

As in the previous section, the update rules of node attitude and the attitude benefit of inter-node game are defined, and the income matrix table is obtained, so according to Table 2, the expected income $E_{\text {active }}$ of the positive attitude node selection propagation information can be calculated as

$$
\begin{aligned}
E_{\text {active } 1} & =\left(\mu_{t}^{i}+R\right) \cdot(1-\gamma)+\mu_{t}^{i} \cdot \gamma \\
& =\mu_{t}^{i}+(1-\gamma) \cdot R
\end{aligned}
$$

The expected income $E_{\text {active } 2}$ that positive attitude node chooses not to disseminate information can be expressed as

$$
\begin{aligned}
E_{\text {active } 2} & =(M+V) \cdot(1-\gamma)+M \cdot \gamma \\
& =M+(1-\gamma) \cdot V
\end{aligned}
$$


Then the positive attitude node selects the propagation with the probability of $\lambda$, and selects the average return of the non-propagating hybrid strategy with the probability of $(1-\lambda)$ to $\bar{E}_{\text {active }}$. Substituting the value of $M$ into the formula, and gets the formula (5)

$$
\begin{aligned}
\bar{E}_{\text {active }} & =\lambda \cdot E_{\text {active } 1}+(1-\lambda) \cdot E_{\text {active } 2} \\
& =\lambda \cdot \mu_{t}^{i}+(1-\gamma) \cdot \lambda R+(1-\lambda) \cdot M+(1-\lambda)(1-\gamma) \cdot V \\
& =\mu_{t}^{i}+(1-\lambda)\left(\mu_{t}^{j}-\mu_{t}^{i}\right) \zeta(i, j)+(1-\gamma)[\lambda(R-V)+V]
\end{aligned}
$$

When the positive attitude node chooses the pure strategy of propagating information, the benefit is higher than the average return of the mixed strategy, then the node will give preference to the strategy, which is a process of positive growth change over time. So according to TAYLOR[15], the earliest proposed method for solving the replication dynamic equation, and combining with the equations (3) and (5), the replication dynamic equations for the positive attitude node selection to propagate information are derived, and expressed as equation (6):

$$
\begin{aligned}
\lambda^{\prime}(t) & =\frac{d \lambda(t)}{d t}=\lambda \cdot\left(E_{\text {active } 1}-\bar{E}_{\text {active }}\right) \\
& =\lambda(1-\lambda)\left(E_{\text {active } 1}-E_{\text {active } 2}\right) \\
& =\lambda(1-\lambda)\left[(R-V)(1-\gamma)-\left(\mu_{t}^{j}-\mu_{t}^{i}\right) \zeta(i, j)\right]
\end{aligned}
$$

Similarly, the expected income $E_{\text {negative }}$ of the non-propagating message in the negative attitude node and the expected income $E_{\text {negativel }}$ of the selected propagating message can be calculated as

$$
\begin{aligned}
E_{\text {negativel }} & =\lambda \cdot \mu_{t}^{j}+(1-\lambda) \cdot\left(\mu_{t}^{j}+J\right) \\
& =\mu_{t}^{j}+(1-\lambda) \cdot J \\
E_{\text {negative } 2} & =\lambda \cdot K+(1-\lambda) \cdot(K-V) \\
& =K-(1-\lambda) \cdot V
\end{aligned}
$$

Then the negative attitude node chooses not to propagate with the probability of $\gamma$, and the average return of the mixed strategy composed of $(1-\gamma)$ probability selection propagation is recorded as $\bar{E}_{\text {negative }}$, and the value of $K$ is substituting into the formula to obtain the formula (9).

$$
\begin{aligned}
\bar{E}_{\text {negative }} & =\gamma \cdot E_{\text {negativel }}+(1-\gamma) \cdot E_{\text {negative } 2} \\
& =\gamma \cdot \mu_{t}^{j}+J+(1-\lambda) \cdot J-\gamma \cdot J \\
& =\mu_{t}^{j}+(1-\gamma) \cdot\left(\mu_{t}^{i}-\mu_{t}^{j}\right) \zeta(i, j)+(1-\lambda)[\gamma \cdot J-(1-\gamma) \cdot V]
\end{aligned}
$$


According to the formula (7) and formula (9), a copy dynamic equation that a negative attitude node does not propagate information can be showed as :

$$
\begin{aligned}
\gamma^{\prime}(t) & =\frac{d \gamma(t)}{d t}=\gamma \cdot\left(E_{\text {negative } 1}-\bar{E}_{\text {negative }}\right) \\
& =\gamma(1-\gamma)\left(E_{\text {negative1 }}-E_{\text {negative } 2}\right) \\
& =\gamma(1-\gamma)\left[(1-\lambda)(J+V)-\left(\mu_{t}^{i}-\mu_{t}^{j}\right) \zeta(i, j)\right]
\end{aligned}
$$

The dynamic replication equations of the joint formula (6) and formula (10), the resulting replication equations are evolutionary game models based on node attitude.

$$
\left\{\begin{array}{l}
\lambda^{\prime}(t)=\frac{d \lambda(t)}{d t} \\
\gamma^{\prime}(t)=\frac{d \gamma(t)}{d t}
\end{array}\right.
$$

In order to obtain the influence of node attitude change on information dissemination, this paper combines the improved SIS dynamics model to analyze the information evolution, and the SIS model includes two state nodes, which are susceptible nodes and propagation nodes, as shown in Figure 1

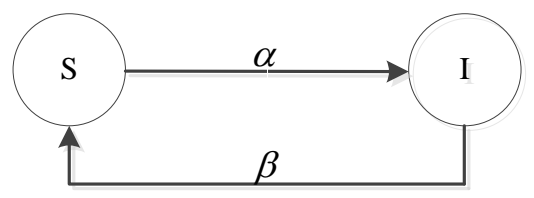

Fig.1. SIS Model Diagram

This corresponds to the two node groups of positive attitude and negative attitude in the social network. During the interaction of the nodes, the attitudes of the two are changed, which may cause the state to change. Assume that the total number of users remains constant for a certain period of time, so the $S(t)+I(t)=1$, and $S(t)$ and $I(t)$ represent the corresponding proportion of positive attitude and negative attitude at a certain moment, respectively. The dynamic equation of the SIS model is as follows:

$$
\left\{\begin{array}{l}
\frac{d S(t)}{d t}=-\alpha S(t) I(t)+\beta I(t) \\
\frac{d I(t)}{d t}=\alpha S(t) I(t)-\beta I(t) \\
S(t)+I(t)=1
\end{array}\right.
$$

In the above model, the game situation between the attitudes of two nodes is discussed. The probability of selection of the forwarding strategy of the positive attitude node and the negative attitude node changes with time until the steady state. Therefore, when a user $i$ interacts with an $m$ neighbor node at a certain point in time, the probability of a change in the state of $k$ nodes satisfies two distribution under the influence of attitude[11]. 


$$
P=(X=k)=\left(\begin{array}{l}
m \\
k
\end{array}\right) \lambda(t)^{k} \gamma(t)^{m-k} \quad(\mathrm{k}=0,1,2 \ldots \mathrm{m})
$$

Then, the probability that a user changes state at a certain moment under the influence of the attitude of the neighbor node is

$$
\delta(t)=\sum_{k=0}^{m} \frac{k}{m}\left(\begin{array}{l}
m \\
k
\end{array}\right) \lambda(t)^{k} \gamma(t)^{m-k} \quad(\mathrm{k}=0,1,2 \ldots \mathrm{m})
$$

The kinetic equation of the formula (12) is rewritten into the state transfer kinetic equation under the influence of attitude, as follows

$$
\left\{\begin{array}{l}
\frac{d S(t)}{d t}=-\delta(t) S(t) I(t)+(1-\delta(t)) I(t) \\
\frac{d I(t)}{d t}=\delta(t) S(t) I(t)-(1-\delta(t)) I(t) \\
S(t)+I(t)=1
\end{array}\right.
$$

\subsection{Evolutionary stability strategy analysis}

Let the dynamic replication equations of equation (11) be equal to zero, and find the equilibrium point of the information propagation evolution game system based on the node attitude. If the obtained solution is the evolutionary stability strategy of the system, use the knowledge of mathematical differential equations to know the available formula (6) and the Jacobian matrix of equation (10) is used to verify each equilibrium point. The Jacobian matrix can obtain the best linear approximation of a differentiable equation at a given fixed point. Under this evolution model, the two parties participate in the game. Whether different attitudes agree on the choice strategy of information dissemination or not. The five equilibrium points are obtained as $\mathrm{A}(0,0), \quad \mathrm{B}(0,1), \quad \mathrm{C}(1,0), \quad \mathrm{D}(1,1)$ and $\mathrm{E}\left(\left(\frac{(J+V)-\left(\mu_{t}^{i}-\mu_{t}^{j}\right) \zeta(i, j)}{J+V}, \frac{(R-V)-\left(\mu_{t}^{j}-\mu_{t}^{i}\right) \zeta(i, j)}{R-V}\right)\right)$. The Jacobian matrix is obtained as follows:

$$
J a c o b i=\left[\begin{array}{cr}
(1-2 \lambda)[(R-V)(1-\gamma) & \\
\left.-\left(\mu_{t}^{j}-\mu_{t}^{i}\right) \zeta(i, j)\right] & \lambda(1-\lambda)(\mathrm{V}-\mathrm{R}) \\
\gamma(\gamma-1)(\mathrm{J}+\mathrm{V}) & (1-2 \gamma)[(J+V)(1-\lambda) \\
& \left.-\left(\mu_{t}^{i}-\mu_{t}^{j}\right) \zeta(i, j)\right]
\end{array}\right]
$$


Therefore, the determinant of the Jacobi matrix is the difference between the main diagonal and the sub-diagonal product, and the trace of the matrix is the sum of the elements on the diagonal, which is calculated and obtained respectively.

$$
\begin{aligned}
& \operatorname{det}(\text { Jacobi })=(1-2 \lambda)\left[(R-V)(1-\gamma)-\left(\mu_{t}^{j}-\mu_{t}^{i}\right) \zeta(i, j)\right] \times \\
&(1-2 \gamma)\left[(J+V)(1-\lambda)-\left(\mu_{t}^{i}-\mu_{t}^{j}\right) \zeta(i, j)-\right. \\
& \lambda(1-\lambda)(\mathrm{V}-\mathrm{R}) \cdot \gamma(\gamma-1)(\mathrm{J}+\mathrm{V}) \\
& \operatorname{tr}(\mathrm{Jacobi})=(1-2 \lambda)\left[(R-V)(1-\gamma)-\left(\mu_{t}^{j}-\mu_{t}^{i}\right) \zeta(i, j)\right]+ \\
&(1-2 \gamma)\left[(J+V)(1-\lambda)-\left(\mu_{t}^{i}-\mu_{t}^{j}\right) \zeta(i, j)\right]
\end{aligned}
$$

For the given equilibrium point is the evolutionary stability strategy solution, according to the nature of the Jacobian matrix, when $\operatorname{det} J>0, t r J<0$, the equilibrium point is the stability strategy point of the evolutionary system. When $\operatorname{det} J>0, \operatorname{tr} J>0$, the equilibrium point is unstable point. When $\operatorname{det} J<0$, the given point is the saddle point regardless of the value of $t r J$. Substituting different points into the equations (17) and (18), find the values of the determinant and trace of the Jacobian corresponding to the equilibrium point. The results are shown in Table 3.

Table 3. The determinant and trace values of the Jacobi matrix corresponding to the different equilibrium points

\begin{tabular}{cll}
\hline $\begin{array}{l}\text { Equilibriu } \\
\text {-m Point }\end{array}$ & \multicolumn{1}{c}{$\operatorname{det} J$} & \multicolumn{1}{c}{$t r J$} \\
\hline$(0,0)$ & {$\left[(R-V)-\left(\mu_{t}^{j}-\mu_{t}^{i}\right) \zeta(i, j)\right] \times$} & \\
& {$\left[(J+V)-\left(\mu_{t}^{i}-\mu_{t}^{j}\right) \zeta(i, j)\right]$} & $R+J$ \\
$(0,1)$ & {$\left[(J+V)-\left(\mu_{t}^{i}-\mu_{t}^{j}\right) \zeta(i, j)\right] \times$} & $2\left(\mu_{t}^{i}-\mu_{t}^{j}\right) \zeta(i, j)-$ \\
& $\left(\mu_{t}^{j}-\mu_{t}^{i}\right) \zeta(i, j)$ & $(J+V)$ \\
$(1,0)$ & {$\left[(R-V)-\left(\mu_{t}^{j}-\mu_{t}^{i}\right) \zeta(i, j)\right] \times$} & $2\left(\mu_{t}^{j}-\mu_{t}^{i}\right) \zeta(i, j)-$ \\
& $\left(\mu_{t}^{i}-\mu_{t}^{j}\right) \zeta(i, j)$ & $(R-V)$ \\
$(1,1)$ & $\left(\mu_{t}^{j}-\mu_{t}^{i}\right)\left(\mu_{t}^{i}-\mu_{t}^{j}\right) \zeta(i, j)$ & 0 \\
\hline
\end{tabular}

$R$ and $J$ are the gains obtained when the positive attitude node and the negative attitude node change the attitude of the other party unilaterally, and $V$ is the benefit when the active node and the negative node change each other's attitude. Since the initial value of the node's attitude and the node's attitude return value are different under different circumstances, the stability of the equilibrium point may also be different. The stability of the equilibrium point will be discussed below for different values.

Scenario 1 When $R>|J|>V$, indicating that a positive attitude node occupies a more dominant position and will actively advocate negative attitude node to spread the message, so the corresponding positive attitude node to the promotion of information dissemination of the 
benefits will be greater than the negative attitude of the node to the information of the inhibitory effect. According to the relationship of attitude value incomes, the stability of different equilibrium points of evolutionary game model can be analyzed, and the discrimination is shown in table 4.

able 4. Stability discrimination of equilibrium points in scenario 1

\begin{tabular}{cccl}
\hline Equilibrium Point & $\operatorname{det} J$ & $t r J$ & Stability \\
\hline$(0,0)$ & - & + & Saddle Point \\
$(0,1)$ & + & + & Unstable point \\
$(1,0)$ & + & - & Stable Point \\
$(1,1)$ & - & 0 & Saddle Point \\
\hline
\end{tabular}

As shown in table 4 , only the equilibrium point $(1,0)$ is a stable point, that is, the evolution game result between the attitude nodes will converge to that point, and the positive attitude node will eventually promote the negative attitude node to the message propagation. Section 2 interaction analysis shows that the positive attitude node changes the willingness of the negative attitude node to spread, and promotes the dissemination of information, and the result is in line with the actual situation.

Scenario 2 When $|J|>R>V$, it means that the negative attitude node dominates the information dissemination at the initial moment, then the negative attitude node will try to change the positive attitude of the positive attitude to the message, thus restraining the information transmission to a certain extent. Through the calculation of the determinant and trace of the Jacobian matrix, as shown in Table 5, the evolutionary game model is finally stable at point $(1,0)$, indicating that the negative attitude node cannot completely suppress the information transmission. As time goes on, eventually some of the information will be circulated on the network.

Table 5. Stability discrimination of equilibrium points in case 2

\begin{tabular}{cccl}
\hline Equilibrium Point & $\operatorname{det} J$ & $t r J$ & Stability \\
\hline$(0,0)$ & - & - & Saddle Point \\
$(0,1)$ & + & + & Unstable point \\
$(1,0)$ & + & - & Stable Point \\
$(1,1)$ & - & 0 & Saddle Point \\
\hline
\end{tabular}

Combining with the stability analysis of equilibrium points in the above two cases, we know that in the initial moment, both positive attitude node and negative attitude dominate the information dissemination, and the equilibrium point $(0,1)$ is the unstable point, that is, when the two attitude nodes interact, in combination with their own evaluation of the information and the influence of the attitude from the neighbor node, attitudes may change, but in the end it is less likely to completely inhibit the spread of information. The same analysis shows that the equilibrium points $(0,0)$ and $(1,1)$ are saddle points, which are physically stable in one direction, but unstable in the other direction, meaning that both attitudes are In the process of interactive game, we want to change with the least attitude to get the maximum benefit, so 
there is no result of not changing the other party's strategy or swapping the strategy. Since equilibrium point $\mathrm{E}$ is an indeterminate point, as defined in section 2 nd above, the initial value of the attitude of different nodes has positive and negative points, so it is not difficult to analyze the value of the $\mathrm{E}$ point is greater than 1 regardless of the value of the $\mathrm{A}, \mathrm{B}$ and $\mathrm{C}$, so the equilibrium point does not exist under the evolutionary system.

\section{Numerical analysis and Simulation experiment of model}

\subsection{Numerical analysis of the model}

In order to verify the validity of evolution game model of the information propagation proposed above, this paper first analyzes the value of the proposed model on MATLAB. According to different situations, the evolution trend of the probability of the message forwarding strategy selection by different attitude nodes is verified.

When $R>|J|>V$, we take the positive attitude node to select the initial probability of propagation that is 0.7 and 0.9 , take the negative attitude node to choose the probability of non-propagation strategy that is 0.3 and 0.1 , and randomly take the attitude convergence parameter that is $\zeta(i, j)=0.5$. The numerical analysis results as shown in Figure 2. At this time, the probability of positive attitude node selection to propagate information approaches 1 with the change of time, and the negative attitude node approaches 0 under the influence of positive attitude node. These indicate that the positive attitude node plays a role in promoting information, which is consistent with the stability strategy analysis of cases 1 of 3.3. In the case of different initial attitude strengths, it can be seen from the numerical results that the system reaches a stable state more quickly with the initial attitude tendency of the positive attitude node is stronger and the negative attitude tendency does not spread weaker. It indicates that the stronger the positive attitude, the more it can promote the dissemination of information.
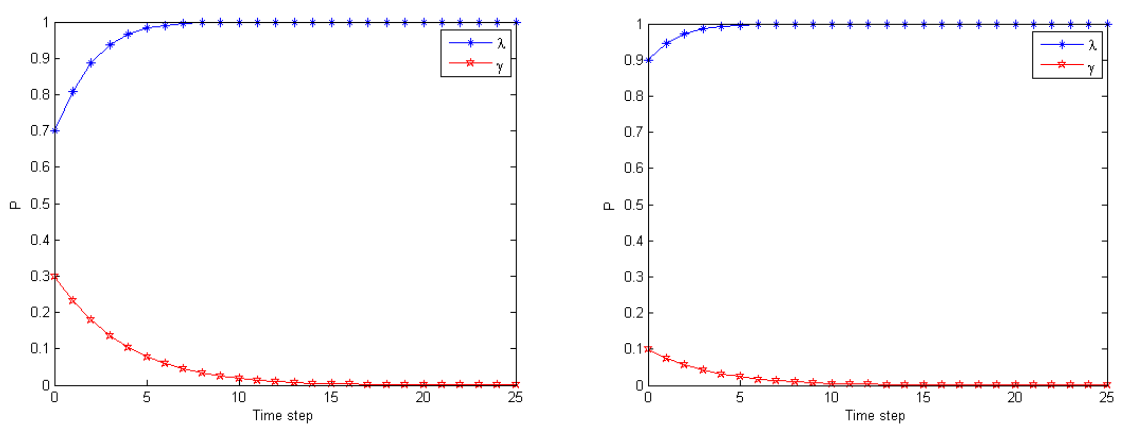

Fig.2. Scenario 1 node Propagation strategy selection probability change trend

When $|J|>R>V$, the negative attitude node dominates at this time, and the relevant parameter values are set as above. At this time, the probability of negative attitude node initial non-selection of information propagation is 0.7 and 0.9 , and the probability of positive attitude node selection of information dissemination is 0.3 and 0.1 . Numerical analysis results as shown in Figure 3, the early negative attitude node reduces the probability that the positive 
attitude node propagates information, to a certain extent which inhibits the dissemination of information, and the attitude of negative attitude node which does not spread information is higher. With the increase of time, the tendency of positive attitude node to spread information will gradually strengthen. So the negative attitude node can not suppress the spread of information all the time, it is more in line with the situation of information communication in the actual network. And then the attitude tendency of negative nodes not to propagate information decreases gradually, which conforms to the stability strategy analysis cases 2 of 3.3. The greater the initial intensity of the negative attitude node, the longer the suppression information propagation time, and the longer it takes to reach the system stabilization time.
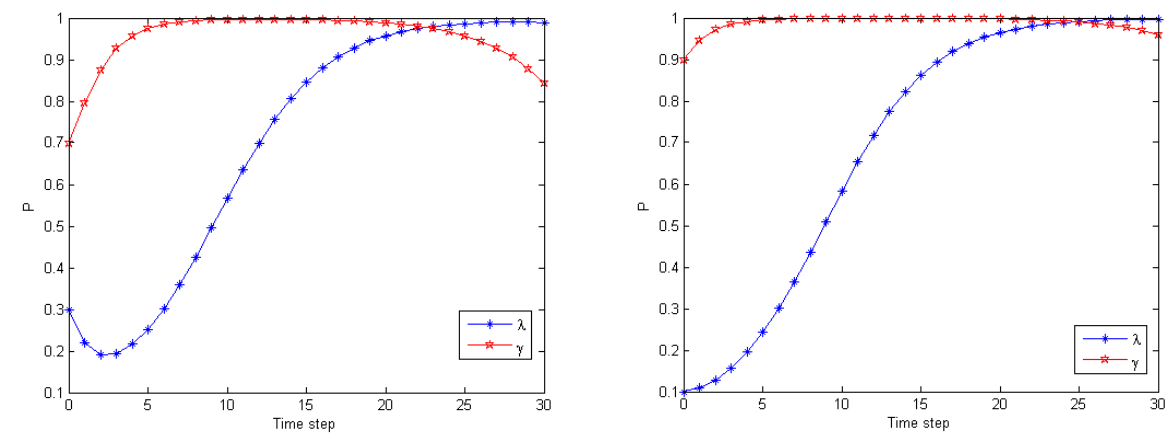

Fig.3. Scenario 2 node Propagation strategy selection probability change trend

In this paper, the attitude convergence parameters are introduced, which indicates the speed of attitude change that the attitude of a node is affected by the attitude of the neighbor node. And it also reflects the speed of the renewal of the attitude of information dissemination, which has an important position of influence. Therefore, when the other parameters are set unchangedly, different attitude convergence parameters are taken to verify the change of probability when the information strategy is selected for the node. As shown in Figure 4, when the convergence parameters are larger, the time of the active node coming to a stable state is shorter, and the convergence degree of the visible node attitude plays an important role in the dissemination of information. Under the positive attitude, the faster convergence of attitudes can promote the speed of information dissemination.

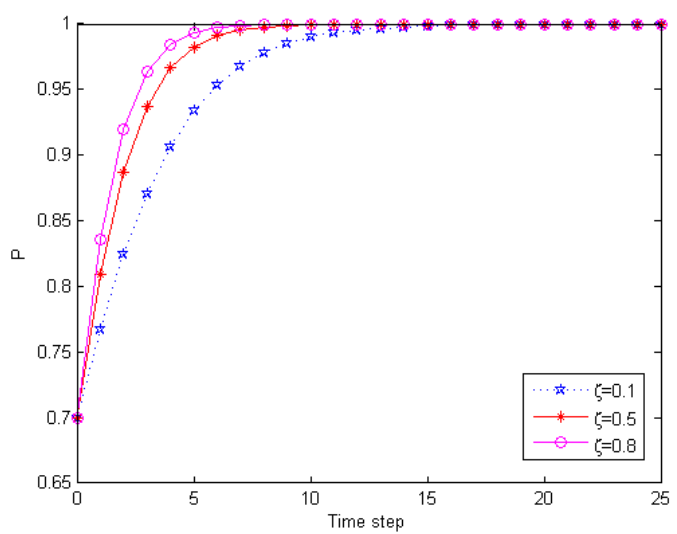

Fig.4. The effect of different convergence parameters on $\lambda$ 
As can be seen from Figure 5, for the negative attitude node, the smaller the attitude convergence parameter, the greater the probability that the node chooses not to propagate the information, and the longer the duration. With the increase of the convergence parameters, the shorter the inhibition time of the negative node to the information, the less the inhibition of the information. The size of the convergence parameters of the attitude has an important influence on the nodes of different attitudes, which is positively correlated with the promotion of positive attitude nodes and negatively correlated with the inhibitory effect of the negative attitude nodes.

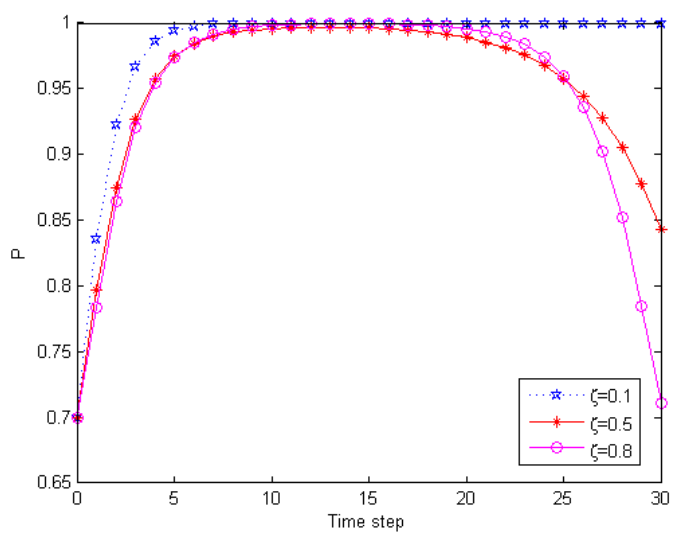

Fig.5. The effect of different convergence parameters on $\gamma$

\subsection{Data simulation}

In order to verify the validity of the model, this paper selects the data set of Sina Weibo to verify the model,which includes the user's behaviors such as forwarding and commenting on hot events. The dataset was collected from September 2014 to March 2015, including twitter contents 40005242, which was forwarded or published by 92176 users, and contains information on Weibo topics with 616 different keywords with attitude tags [16]. This article selected the "Double 11" that involved the user and message forwarding relationship as a dataset, which started from November 4, 2014 to November 18.The total number of nodes are 312589 , and the number of edges are 835862 .The average degree is 2.674 , and the average clustering coefficient 0.094 . This paper mainly carries out the data simulation analysis from two aspects, the first one is to study the change of S and I nodes in the model. And the second one is to verify the change trend of information dissemination in the networks that influenced by positive and negative attitude nodes when taking different attitude convergence parameters, so as to analyze the effects of node attitudes on information dissemination.

As shown in Figure 6, in the initial state, the propagation state nodes are only a minority, and most of the nodes are uninfected. As time progresses, the nodes interact frequently and the state of the nodes changes rapidly in the first half of the period, and then gradually enters a stable state. The uninfected nodes are fewer and fewer with the increase of propagation nodes, but some nodes become non-information status again. Therefore, when the equilibrium is reached, the propagation nodes are less than the initial value of the non-propagation nodes, and the nodes not propagating are more than the initial value of propagation nodes. 


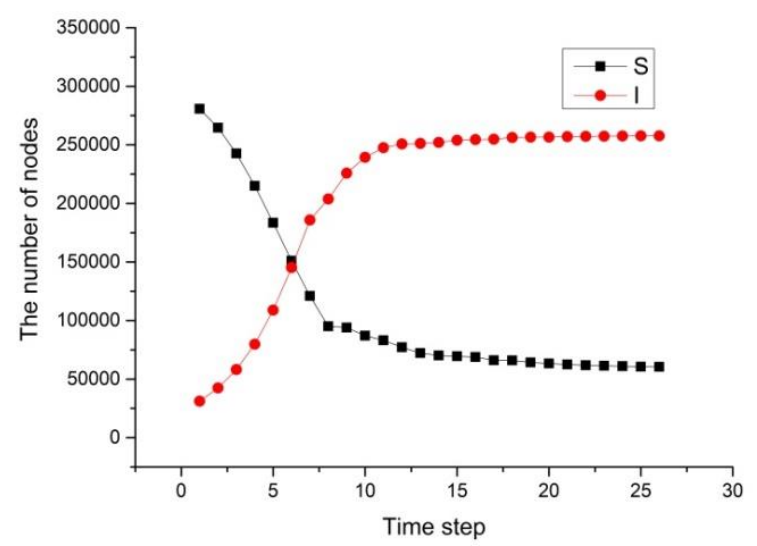

Fig.6. State change curve of S,I node

When the positive attitude dominates in accordance with situation 1, the basic parameters setting as above, the experimental results are shown in Figure 7. The number of positive attitude nodes increases rapidly and reaches the maximum propagation value in a short time. Then, as the timeliness of information gradually decreases, the negative attitude nodes grow more slowly and reach a peak value that is far less than the positive attitude nodes, and finally approaches 0 . This proves the correctness of the numerical theoretical analysis results, and the positive attitude nodes have a greater effect on the dissemination of information.

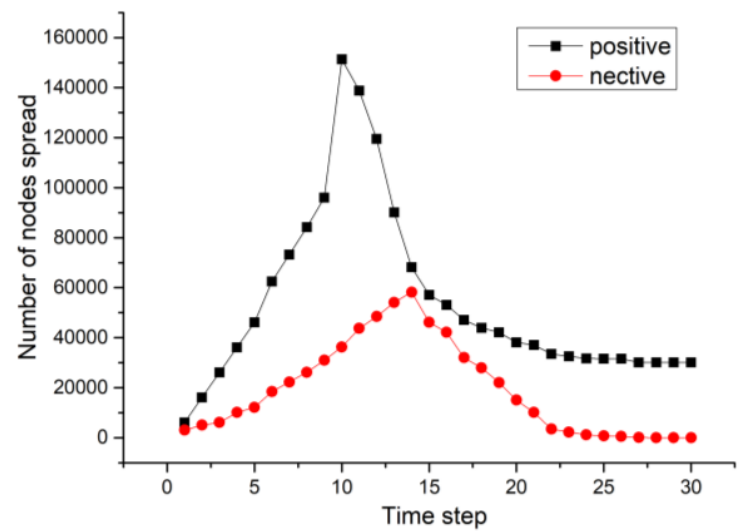

Fig.7. Scenario 1 Propagation node change of different attitudes

In the above scenario 2, as shown in Figure 8, when the negative attitude of the initial stage dominates, the number of nodes with the negative attitude increases rapidly and reaches the maximum value earlier than the positive attitude nodes. At this time, the negative attitude node has the strongest ability to suppress information dissemination, while the positive attitude nodes are slow to grow early due to the influence of the negative attitude nodes. However, the passive nodes gradually decrease. At the same time, the positive attitude nodes will gradually increase and gradually decrease after reaching the maximum value, but the final approaching stability value is greater than the number of negative attitude nodes. That is because some of the information will continue to spread in the networks, in line with the actual network propagation law. 


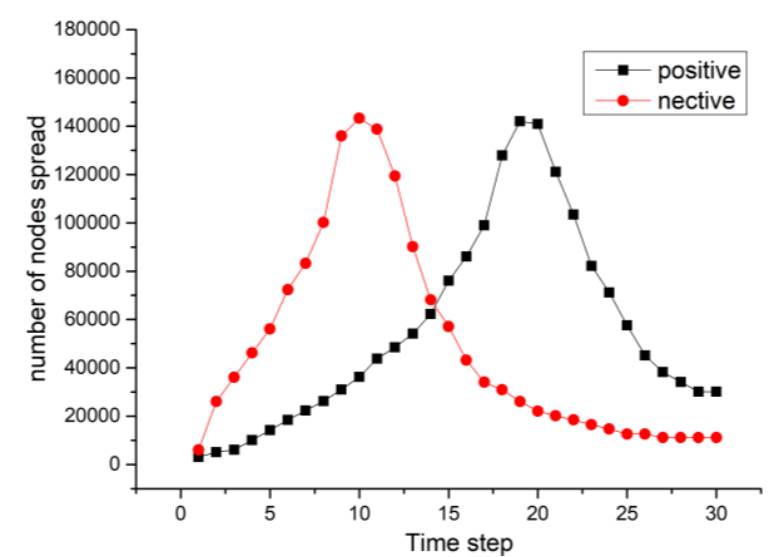

Fig.8. Scenario 2 propagation node change of different attitudes

When the convergence parameters of different attitudes are taken, as shown in Figure 9, it is obvious that the larger the convergence parameters are, the faster the information is propagated in the networks, and the more the numbers of propagation, the longer the duration. As analyzed in the foregoing, the greater the convergence parameters, the faster the positive attitude nodes reache stability. For negative attitude nodes, the smaller the convergence parameter value, the stronger ability to suppress information propagation.

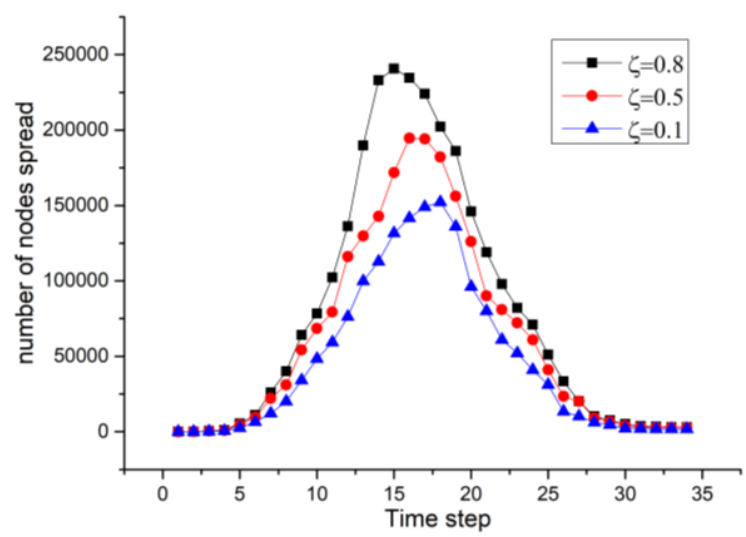

Fig.9. Effects of convergence parameters of different attitudes on information dissemination

\section{Conclusions}

This paper presents a information dissemination model based on evolutionary game and the attitude of nodes in the social networks. When considering the influences of node's attitude, we define the update rule of node attitude according to the non-Bayesian social learning method. Then the evolutionary game model based on the attitude interaction of different nodes is established, and the equilibrium points of the evolutionary system are obtained by the dynamic equation of the replicator. The local stability of each equilibrium point is analyzed by the Jacobi matrix, and the evolutionary stability strategy points under 
different conditions are obtained. Finally, the validity and correctness of the model are verified by numerical analysis and simulation experiments. Experiments show that the model can well describes the information dissemination trend between different attitude nodes in the networks that also influenced by the interaction between them.

Funding: This work was supported in part by the National Natural Science Foundation of China under Grant No. 61871062, and in part by the Scientific Research Foundation of CQUPT under Grant No. A2018-07. 


\section{References}

[1] Huang H C, Lai L C, Hu M, et al.: Information propagation control method in social networks based on exact controllability theory.Journal of electronics \& information technology. 40(7): 17071714(2018)

[2] Li T, Wang Y, Guan Z H. Spreading dynamics of a SIQRS epidemic model on scale-free networks. Communications in Nonlinear Science and Numerical Simulation. 19(3): 686-692(2014).

[3] Wang S, Cheng W, Hao Y.: Designing efficient hybrid strategies for information spreading in scale-free networks. Royal Society open science. 5(8): 180117 (2018).

[4] Gaeta R.: A Model of Information Diffusion in Interconnected Online Social Networks. ACM Transactions on the Web (TWEB). 12(2): 13 (2018).

[5] Zhou T , Liu J G , Bai W J , et al.: Behaviors of susceptible-infected epidemics on scale-free networks with identical infectivity. Physical Review E. 74(5):056109 (2006).

[6] Kang H, Fu X.: Epidemic spreading and global stability of an SIS model with an infective vector on complex networks. Communications in Nonlinear Science \& Numerical Simulation. 27(1-3):30-39 (2015).

[7] Zhao L, Wang J , Chen Y, et al.: SIHR rumor spreading model in social networks. Physica A Statistical Mechanics \& Its Applications. 391(7):2444-2453 (2012).

[8] Wang H ,Han J H, Deng L et al.: Dynamics of rumor spreading in mobile social networks. Acta Phys. Sin. 62(11): 110505(2013).

[9] Zhao N , Cui X.: Impact of individual interest shift on information dissemination in modular networks. Physica A Statistical Mechanics \& Its Applications. 466:232-242 (2017).

[10] Zan Y, Wu J , Li P , et al.: SICR rumor spreading model in complex networks: Counterattack and self-resistance. Physica A Statistical Mechanics \& Its Applications. 405(405):159-170 (2014).

[11] Xiao Y P, Li S Y ,Liu Y B.: An information diffusion dynamic model based on social influence and mean-field theory[J]. Acta Physica Sinica. 66(3) (2017).

[12] Liu X, He D, Yang L, et al.:A novel negative feedback information dissemination model based on online social network. Physica A: Statistical Mechanics and its Applications. 513: 371-389 (2019).

[13] Huang J M, Zhang H W.: A method for selecting defense strategies based on stochastic evolutionary game model. Acta electronica sinica.46(9): 2222-2228(2018).

[14] Huang H C,Sun S R,Hu M.: An information diffusion model of social network based on node attitude. Advanced engineering science.50(01):113-119.(2018).

[15] Karataev E, Zadorozhny V.: Adaptive social learning based on crowdsourcing. IEEE Transactions on Learning Technologies.10(2):128-139(2017).

[16] Taylor P, Jonker L.: Evolutionary stable strategies and game dynamics. Mathematical Biosciences. 40(1-2):145-156 (1978).

[17] Fan R, Xu K, Zhao J.: Higher contagion and weaker ties mean anger spreads faster than joy in social media. arXiv preprint. arXiv:1608.03656 (2016). 